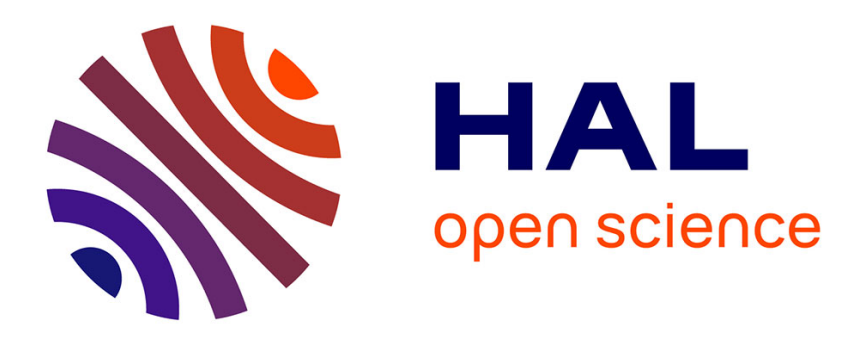

\title{
Configurational Forces in the Theory of Two-Phase Plates
}

\author{
V. A. Eremeyev, Holm Altenbach
}

\section{To cite this version:}

V. A. Eremeyev, Holm Altenbach. Configurational Forces in the Theory of Two-Phase Plates. 2009, pp.121-130. hal-00830609

\section{HAL Id: hal-00830609 \\ https://hal.science/hal-00830609}

Submitted on 5 Jun 2013

HAL is a multi-disciplinary open access archive for the deposit and dissemination of scientific research documents, whether they are published or not. The documents may come from teaching and research institutions in France or abroad, or from public or private research centers.
L'archive ouverte pluridisciplinaire HAL, est destinée au dépôt et à la diffusion de documents scientifiques de niveau recherche, publiés ou non, émanant des établissements d'enseignement et de recherche français ou étrangers, des laboratoires publics ou privés. 


\title{
Configurational Forces in the Theory of Two-Phase Plates
}

Victor A. Eremeyev and Holm Altenbach

\begin{abstract}
Within the framework of the direct approach to the theory of plates the configurational forces are introduced taking into account the transverse shear stiffness. Their applications to plates made of non-homogeneous materials such as laminates, functionally graded materials, two-phase materials, etc., are discussed. The basic items of the applied theory of plates are related to the formulation of all balances for a deformable directed surface and to the specification of the constitutive equations. The variational principle of the total energy is established for plates assuming the presence of a variable surface singular curve. The surface singular curve separates the parts of the plate with different material properties. The continuity conditions at the singular curve are obtained. The energy-momentum tensor is constructed. Equilibrium and quasistatic motion of the surface singular curve are investigated on the base of the proposed kinetic equation. As an example of application of configurational forces we present the model of the deformation of a plate made of a material undergoing phase transitions (e.g., a shape memory alloy).
\end{abstract}

\section{Introduction}

The concept of the configurational force is useful in continuum mechanics as was shown in $[13,18,20,26,29]$ among others. Configuration forces arise from the consideration of motion of defects in the continuous medium. Generally, the nature of defects may be different. Here we consider a defect as a singular curve which separates the parts of a plate consisting of different phases that means, we consider the equilibrium of plates made of materials undergoing phase transitions.

Victor A. Eremeyev

South Scientific Center of RASci \& South Federal University, Milchakova str. 8a,

344090 Rostov on Don, Russia; e-mail: eremeyev.victor@gmail.com

Holm Altenbach

Martin-Luther-Universität Halle-Wittenberg, D-06099 Halle (Saale), Germany;

e-mail: holm.altenbach@iw.uni-halle.de 
The phase transitions (PT) of martensitic type are very important in the mechanics of materials. In particular, PT are responsible for the shape memory effect in some materials, see, e.g., [8,23]. Mechanics of solids with PT has been developed in a number of papers and books, see for instance [1, 7, 8, 16-18, 23, 24].

The growing interest to understanding of the behavior of such two-dimensional structures as thin films, plates and shells made of shape memory alloys and other materials undergoing PT is based on the perspective applications of these structures for design of microelectromechanical systems (MEMS). The mechanics of martensititic thin films was discussed, for example, in $[8,9,19]$. Let us note that experiments on shape memory alloys are usually performed with thin-walled samples, for example rectangular plates $[8,12,27]$.

Within the dynamically and kinematically exact theory of shells presented in $[11,25]$, the equilibrium conditions as well as the continuity conditions for quasistatic deformations of elastic thin-walled structures (plates and shells) undergoing PT of the martensitic type were formulated in $[14,15,28]$. By analogy to the threedimensional case, in the two-dimensional theory of shells a singular surface curve was taken as the phase boundary in the shell. For the two-phase shells the Eshelby's (energy-momentum) tensor was introduced in [14]. From the point of view of configurational mechanics this tensor represents the configurational (or driving) force acting on the phase interface. Thus, the tensor is responsible for its motion. The configurational forces were investigated considering the theory of elastic beams in [20-22] while for the elastic plates in [10,20], respectively.

Following $[14,28]$ we obtain here the equilibrium conditions at the phase interface applying the 5-parametric theory of plates presented in [4-6]. The two-phase plate is considered as some material surface consisting of two material phases divided by a sufficiently smooth surface curve. Below the following items are discussed. Considering [4-6, 30,31] in Section 2 we recall the governing equations of the linear theory of plates based on the direct approach. Here we also introduce both the static and the kinematic compatibility conditions at the curvilinear phase boundary. In Section 3 we formulate the variational principle of stationarity of the total energy functional taking into account the variable surface singular curve. From the variational principle we deduce the additional thermodynamic equilibrium continuity condition satisfied at the phase interface curve. This condition is written using the energy-momentum tensor $\boldsymbol{\mu}$. In Section 4 we assume the kinetic equation describing the motion of the phase interface curve during the quasistatic deformation processes of the two-phase plate. As an example the tension of a two-phase rectangular plate is briefly discussed in Section 5 .

\section{Basic Equations of the Direct Theory of Plates}

Let us assume the geometrically and physically linear plate theory based on the socalled direct approach. In this case one states a two-dimensional deformable surface. On each part of this deformable surface forces and moments are acting - they are 
Fig. 1 Two-phase plate

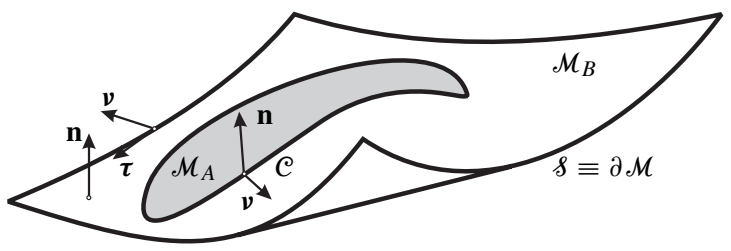

the primary variables. The next step is the introduction of the deformation measures. Finally, it is necessary to interlink the forces and the moments with the deformation variables (constitutive equations). Such a plate theory is formulated by a more natural way in comparison with the other approaches because it is so strong and so exact as the three-dimensional continuum mechanics. But the identification of the stiffness and other parameters is a non-trivial problem and must be realized for each class of plates individually.

In the considered theory of plates we make two basic assumptions:

Assumption 1: The plate (homogeneous or inhomogeneous in transverse direction) can be represented by a deformable surface $\mathcal{M}$ (Figure 1).

Assumption 2: $\quad$ Each material point is an infinitesimal rigid body with 5 degrees of freedom ( 3 translations and 2 rotations).

In addition, the theory presented here is limited by small displacements and rotations and the quadratic strain energy density assumptions.

The equilibrium equations and the kinematic equations are given by the relations $[4-6,30,31]$

$$
\begin{gathered}
\nabla \cdot \mathrm{T}+\mathbf{q}=\mathbf{0}, \quad \nabla \cdot \mathrm{M}+\mathrm{T}_{\times}+\mathbf{m}=\mathbf{0}, \\
\boldsymbol{\epsilon}=\frac{1}{2}\left[\nabla \mathbf{v}+(\nabla \mathbf{v})^{\mathrm{T}}\right], \quad \boldsymbol{\gamma}=\nabla w+\mathrm{c} \cdot \boldsymbol{\varphi}, \quad \boldsymbol{\kappa}=\nabla \boldsymbol{\varphi} .
\end{gathered}
$$

Here T, $\mathrm{M}$ are the tensors of forces and moments, $\mathbf{q}, \mathbf{m}$ are the surface load vectors (forces and moments), $T_{\times}$is the vector invariant of the force tensor, $\nabla$ is the nabla operator, $\mathbf{v}=\mathbf{u} \cdot \mathrm{a}, w=\mathbf{u} \cdot \mathbf{n}, \mathbf{u}, \boldsymbol{\varphi}$ are the vectors of displacements and rotations, and $(\ldots)^{\mathrm{T}}$ denotes transposed. $\mathbf{a}$ is the first metric tensor, $\mathbf{n}$ is the unit normal vector, $\mathbf{c}=-\mathbf{a} \times \mathbf{n}$ is the discriminant tensor, $\boldsymbol{\epsilon}, \boldsymbol{\gamma}$ and $\boldsymbol{\kappa}$ are the tensor of in-plane strains, the vector of transverse shear strains and the tensor of the out-of-plane strains, respectively. In the case of an orthotropic material behavior and a plane mid-surface we assume the following strain energy and constitutive equations

$$
\begin{aligned}
W(\boldsymbol{\epsilon}, \boldsymbol{\gamma}, \boldsymbol{\kappa}) & =\frac{1}{2} \boldsymbol{\epsilon} \cdot \cdot \mathrm{A} \cdot \boldsymbol{\epsilon}+\boldsymbol{\epsilon} \cdot \mathrm{B} \cdot \boldsymbol{\kappa}+\frac{1}{2} \boldsymbol{\kappa} \cdot \cdot \mathrm{C} \cdot \boldsymbol{\kappa}+\frac{1}{2} \boldsymbol{\gamma} \cdot \Gamma \cdot \boldsymbol{\gamma} \\
& +\mathrm{N}_{0} \cdot \boldsymbol{\varepsilon}+\mathrm{M}_{0}^{\mathrm{T}} \cdot \boldsymbol{\kappa}+\mathbf{Q}_{0} \cdot \boldsymbol{\gamma}+W_{0}, \\
\mathrm{~N} \equiv \mathrm{T} \cdot \mathrm{a} & =\frac{\partial W}{\partial \boldsymbol{\epsilon}}, \quad \mathbf{Q} \equiv \mathrm{T} \cdot \mathrm{n}=\frac{\partial W}{\partial \boldsymbol{\gamma}}, \quad \mathrm{M}^{\mathrm{T}}=\frac{\partial W}{\partial \boldsymbol{\kappa}} .
\end{aligned}
$$

A, B, C are 4th rank tensors, $\Gamma$ is a 2 nd rank tensor expressing the effective stiffness properties. They depend on the material properties and the cross-section geometry 
and given by the relations [31]

$$
\begin{aligned}
& \mathrm{A}=A_{11} \mathrm{a}_{1} \mathrm{a}_{1}+A_{12}\left(\mathrm{a}_{1} \mathrm{a}_{2}+\mathrm{a}_{2} \mathrm{a}_{1}\right)+A_{22} \mathrm{a}_{2} \mathrm{a}_{2}+A_{44} \mathrm{a}_{4} \mathrm{a}_{4}, \\
& \mathrm{~B}=B_{13} \mathrm{a}_{1} \mathrm{a}_{3}+B_{14} \mathrm{a}_{1} \mathrm{a}_{4}+B_{23} \mathrm{a}_{2} \mathrm{a}_{3}+B_{24} \mathrm{a}_{2} \mathrm{a}_{4}+B_{42} \mathrm{a}_{4} \mathrm{a}_{2}, \\
& \mathrm{C}=C_{22} \mathrm{a}_{2} \mathrm{a}_{2}+C_{33} \mathrm{a}_{3} \mathrm{a}_{3}+C_{34}\left(\mathrm{a}_{3} \mathrm{a}_{4}+\mathrm{a}_{4} \mathrm{a}_{3}\right)+C_{44} \mathrm{a}_{4} \mathrm{a}_{4}, \\
& \Gamma=\Gamma_{1} \mathrm{a}_{1}+\Gamma_{2} \mathrm{a}_{2},
\end{aligned}
$$

where $a_{1}=a=\mathbf{e}_{1} \mathbf{e}_{1}+\mathbf{e}_{2} \mathbf{e}_{2}, a_{2}=\mathbf{e}_{1} \mathbf{e}_{1}-\mathbf{e}_{2} \mathbf{e}_{2}, a_{3}=c=\mathbf{e}_{1} \mathbf{e}_{2}-\mathbf{e}_{2} \mathbf{e}_{1}, a_{4}=$ $\mathbf{e}_{1} \mathbf{e}_{2}+\mathbf{e}_{2} \mathbf{e}_{1}$, and $\mathbf{e}_{1}, \mathbf{e}_{2}$ are unit basis vectors of an orthonormal coordinate system. In addition, one obtains the orthogonality condition for the $\mathrm{a}_{i}(i=1,2,3,4)$

$$
\frac{1}{2} \mathrm{a}_{i} \cdot \mathrm{a}_{j}=\delta_{i j},
$$

where.. is the double inner (dot) product. $\mathrm{N}_{0}, \mathrm{M}_{0}$ are the tensors of the initial inplane forces and moments, $\mathbf{Q}_{0}$ is the vector of the initial shear forces, while $W_{0}$ is the initial value of the strain energy.

The identification of the effective stiffness tensors A, B, C and $\Gamma$ should be performed on the base of the properties of the real material. Let us assume the Hooke's law with material properties which depend on the normal coordinate $z$. The identification of the effective properties can be performed with the help of static boundary value problems (two-dimensional, three-dimensional) and the comparison of the forces and moments (in the sense of averaged stresses or stress resultants). Finally, we get the following expressions for the classical stiffness tensor components [2-4]

$$
\begin{array}{ll}
\left(A_{11} ;-B_{13} ; C_{33}\right) & =\frac{1}{4}\left\langle\frac{E_{1}+E_{2}+2 E_{1} v_{21}}{1-v_{12} v_{21}}\left(1 ; z ; z^{2}\right)\right\rangle, \\
\left(A_{22} ; B_{24} ; C_{44}\right) & =\frac{1}{4}\left\langle\frac{E_{1}+E_{2}-2 E_{1} v_{21}}{1-v_{12} v_{21}}\left(1 ; z ; z^{2}\right)\right\rangle, \\
\left(A_{12} ;-B_{23}=B_{14} ;-C_{34}\right) & =\frac{1}{4}\left\langle\frac{E_{1}-E_{2}}{1-v_{12} \nu_{21}} z\left(1 ; z ; z^{2}\right)\right\rangle, \\
\left(A_{44} ;-B_{42} ; C_{22}\right) & =\left\langle G_{12}\left(1 ; z ; z^{2}\right)\right\rangle,
\end{array}
$$

where $\langle\ldots\rangle$ is the integral over the plate thickness $h$, while $E_{1}, E_{2}, v_{12}, v_{21}, G_{12}$ are the elastic moduli of the orthotropic bulk material. In addition, two non-classical stiffness are obtained

$$
\Gamma_{1}=\frac{1}{2}\left(\lambda^{2}+\eta^{2}\right) \frac{A_{44} C_{22}-B_{42}^{2}}{A_{44}}, \quad \Gamma_{2}=\frac{1}{2}\left(\eta^{2}-\lambda^{2}\right) \frac{A_{44} C_{22}-B_{42}^{2}}{A_{44}} .
$$

Here $\eta^{2}$ and $\lambda^{2}$ are the smallest non-zero eigen-values of Sturm-Liouville problems

$$
\frac{\mathrm{d}}{\mathrm{d} z}\left(G_{1 n} \frac{\mathrm{d} Z}{\mathrm{~d} z}\right)+\eta^{2} G_{12} Z=0, \quad \frac{\mathrm{d}}{\mathrm{d} z}\left(G_{2 n} \frac{\mathrm{d} Z}{\mathrm{~d} z}\right)+\lambda^{2} G_{12} Z=0,\left.\quad \frac{\mathrm{d} Z}{\mathrm{~d} z}\right|_{|z|=h / 2}=0 .
$$

The boundary conditions are given by 


$$
\boldsymbol{v} \cdot \mathbf{T}=\mathbf{f}, \quad \boldsymbol{v} \cdot \mathrm{M}=\mathbf{l}, \quad(\mathbf{l} \cdot \mathbf{n}=0) \quad \text { along } \wp_{f},
$$

and

$$
\mathbf{u}=\mathbf{u}^{0}, \quad \boldsymbol{\varphi}=\varphi^{0} \quad \text { along } \diamond_{u} .
$$

Here $\mathbf{f}$ and $\mathbf{I}$ are external force and couple vectors acting along the part $S_{f}$ of the boundary of the plate $\delta=\varsigma_{f} \cup s_{u} \equiv \partial \mathcal{M}$, while $\mathbf{u}^{0}$ and $\varphi^{0}$ are given functions describing the displacements and rotation of the plate boundary $\delta_{u}$, respectively. $\boldsymbol{v}$ is the unit normal vector to $\delta(\boldsymbol{v} \cdot \mathbf{n}=0)$. The relations (8) and (9) are the static and kinematic boundary conditions, respectively. Other mixed types of boundary conditions are possible. For example, the simple support boundary conditions corresponding to a hinge are given by

$$
\boldsymbol{v} \cdot \mathrm{M} \cdot \boldsymbol{\tau}=\mathbf{0}, \quad \mathbf{u}=\mathbf{0}, \quad \boldsymbol{\varphi} \cdot \boldsymbol{\tau}=0 .
$$

Here $\boldsymbol{\tau}$ is the unit tangent vector to $\&(\boldsymbol{\tau} \cdot \mathbf{n}=\boldsymbol{\tau} \cdot \boldsymbol{v}=0)$.

Let us consider the two-phase plate consisting of a material undergoing the phase transformations. In this case the plate is modeled by the surface $\mathcal{M}=\mathcal{M}_{A} \cup \mathcal{M}_{B}$, where $\mathcal{M}_{A, B}$ are surfaces consisting of phases $A$ and $B$, respectively. The phase interface is a smooth curve $\mathcal{C}$ separating the surfaces $\mathcal{M}_{A, B}$ (Figure 1).

The curvilinear phase interfaces in plates can be either coherent or incoherent in rotations, see [14]. For the coherent interface both fields $\mathbf{u}$ and $\varphi$ are supposed to be continuous at $\mathcal{C}$

$$
\llbracket \mathbf{u} \rrbracket=\mathbf{0}, \quad \llbracket \varphi \rrbracket=\mathbf{0},
$$

where double square brackets denote a jump of discontinuity across $\mathcal{C}: \llbracket(\ldots) \rrbracket=$ $(\ldots)_{B}-(\ldots)_{A}$.

The phase interface is called incoherent in rotations if only $\mathbf{u}$ is continuous at $\mathcal{C}$ but the continuity of $\varphi$ may be violated. In this case the condition $(10)_{1}$ is still satisfied, but $(10)_{2}$ may be violated.

\section{Variation Principle of Total Energy}

Following [14] we obtain the phase equilibrium conditions along the phase interface curve $\mathcal{C}$ using the variational principle

$$
\delta E=0, \quad E=\int_{\mathcal{M}_{A}} W_{A} d a+\int_{\mathcal{M}_{B}} W_{B} d a-A^{\mathrm{ext}},
$$

where $E$ is the functional of the total energy, $W_{A, B}$ are the strain energies corresponding to the phases $A, B$, and $A^{\text {ext }}$ is the functional of external loads. We assume that $\delta A^{\text {ext }}$ is given by the relation

$$
\delta A^{\mathrm{ext}}=\int_{\mathcal{M} \backslash \mathcal{C}}(\mathbf{q} \cdot \delta \mathbf{u}+\mathbf{m} \cdot \delta \boldsymbol{\varphi}) d a+\int_{\delta_{f}}(\mathbf{f} \cdot \delta \mathbf{u}+\mathbf{l} \cdot \delta \boldsymbol{\varphi}) d s,
$$


where $\delta \mathbf{u}$ and $\delta \varphi$ are variations of $\mathbf{u}$ and $\boldsymbol{\varphi}$, respectively. Let us note that $\delta \mathbf{u}$ and $\delta \boldsymbol{\varphi}$ satisfy the kinematic relations

$$
\delta \mathbf{u}=\mathbf{0}, \quad \delta \boldsymbol{\varphi}=\mathbf{0} \quad \text { along } \varsigma_{u},
$$

as well as the continuity conditions along $\mathcal{C}$. For the coherent interface the continuity conditions are

$$
\llbracket \delta \mathbf{u} \rrbracket+V \llbracket v \cdot \nabla \mathbf{u} \rrbracket=\mathbf{0}, \quad \llbracket \delta \varphi \rrbracket+V \llbracket v \cdot \nabla \varphi \rrbracket=\mathbf{0} .
$$

where $V$ is the virtual velocity of $\mathcal{C}$, see [14], while for the phase interfaces incoherent in rotations the continuity conditions reduce to the relation (14) 1 .

The variation of $E$ is given by

$$
\delta E=\int_{\mathcal{M} \backslash \mathcal{C}} \delta W d a-\int_{\mathcal{C}} V \llbracket W \rrbracket d s-\delta A^{\mathrm{ext}},
$$

where

$$
\delta W=\frac{\partial W}{\partial \boldsymbol{\epsilon}} \cdot \delta \boldsymbol{\epsilon}^{\mathrm{T}}+\frac{\partial W}{\partial \boldsymbol{\gamma}} \cdot \delta \boldsymbol{\gamma}+\frac{\partial W}{\partial \boldsymbol{\kappa}} \cdot \delta \boldsymbol{\kappa}^{\mathrm{T}} .
$$

Using Eqs (13), (14) from Eq. (11) 1 one obtains the equilibrium equations (1), the static boundary conditions (8) as well as the following general continuity condition to be satisfied at the interface $\mathcal{C}$ :

$$
\int_{\mathcal{C}}\{V \llbracket W \rrbracket+\llbracket \boldsymbol{v} \cdot \mathrm{T} \cdot \delta \mathbf{u} \rrbracket+\llbracket \boldsymbol{v} \cdot \mathrm{M} \cdot \delta \boldsymbol{\varphi} \rrbracket\} d s=0 .
$$

The balance equation on the phase interface $\mathcal{C}$ following from Eq. (16) consist of both the static balance equations and the so-called thermodynamical balance equation which is necessary for the determination of $\mathcal{C}$. For the coherent interface the static balance equations are

$$
\boldsymbol{v} \cdot \llbracket \mathrm{T} \rrbracket=\mathbf{0}, \quad \boldsymbol{v} \cdot \llbracket \mathrm{M} \rrbracket=\mathbf{0},
$$

while the thermodynamical balance equation is given by

$$
\boldsymbol{v} \cdot \llbracket \mu_{\mathrm{C}} \rrbracket \cdot v=0 .
$$

For the phase interface incoherent in rotations from the variational principle we obtain the following continuity conditions along $\mathcal{C}$ :

$$
\boldsymbol{v} \cdot \llbracket \mathrm{T} \rrbracket=\mathbf{0}, \quad \boldsymbol{v} \cdot \mathrm{M}_{A, B}=\mathbf{0}, \quad \boldsymbol{v} \cdot \llbracket \mu_{\mathrm{I}} \rrbracket \cdot v=0
$$

Here

$$
\boldsymbol{\mu}_{\mathrm{C}}=W \mathbf{a}-\mathrm{N} \cdot(\nabla \mathbf{v})^{\mathrm{T}}-\mathbf{Q} \nabla w-\mathrm{M}^{\mathrm{T}} \cdot(\nabla \boldsymbol{\varphi})^{\mathrm{T}}, \quad \boldsymbol{\mu}_{\mathrm{I}}=W \mathbf{a}-\mathrm{N} \cdot(\nabla \mathbf{v})^{\mathrm{T}}-\mathbf{Q} \nabla w .
$$


$\boldsymbol{\mu}_{\mathrm{C}}$ and $\boldsymbol{\mu}_{\mathrm{I}}$ are the Eshelby's tensors (the energy-momentum tensors) for the coherent interface and the incoherent one in the two-phase plate, respectively. The condition $\boldsymbol{v} \cdot \llbracket \boldsymbol{\mu} \rrbracket \cdot \boldsymbol{v}=0$ should be satisfied, where $\boldsymbol{\mu}=\boldsymbol{\mu}_{\mathrm{C}}$ for the coherent phase interface and $\boldsymbol{\mu}=\boldsymbol{\mu}_{\mathrm{I}}$ for the incoherent in rotations one. From the point of view of configurational mechanics the quantity $\boldsymbol{v} \cdot \llbracket \boldsymbol{\mu} \rrbracket \cdot \boldsymbol{v}$ represents the configurational (or driving) force acting on $\mathcal{C}$ and responsible for its motion for non-equilibrium deformations.

\section{Kinetic Equation}

Let us consider quasistatic deformation process, where the equilibrium conditions (1), (8) and (17) are satisfied while the $\boldsymbol{v} \cdot \llbracket \mu \rrbracket \cdot v=0$ is not. For the quasi-static process we obtain the formula

$$
\frac{\mathrm{d} E}{\mathrm{~d} t}=-\int_{\mathcal{C}} V F d s, \quad F=\boldsymbol{v} \cdot \llbracket \mu \rrbracket \cdot \boldsymbol{v} .
$$

Here $F$ is the configurational force acting on the phase boundary, while $V$ is the velocity of $\mathcal{C}$. By analogy to three-dimensional case [1] Eq. (19) leads to the kinetic equation describing the propagation of $\mathcal{C}$

$$
V=k(F)
$$

with a non-negative definite kinetic function $k$. In the theory of elasticity the kinetic equations of the type (19) were discussed in a number of papers and monographs, see for example $[1,7,8,17,18,24]$. The equation (19) can also be regarded as a constitutive relation consistent with the thermodynamic requirement $\mathrm{d} E / \mathrm{d} t \leq 0$.

Following [1], let us assume $k(\varsigma)$ in the form

$$
k(F)= \begin{cases}K\left(F-F_{0}\right), & F \geq F_{0}, \\ 0, & -F_{0}<F<F_{0}, \\ K\left(F+F_{0}\right), & F \leq-F_{0} .\end{cases}
$$

Here $F_{0}$ describes effects associated with the nucleation of the new phase and action of the surface tension, see [1], and $K$ is a positive kinetic factor. If $F_{0}=0$ then the function (20) reduces to the linear kinetic function $k(F)=K F$.

\section{Tension of Two-Phase Rectangular Plate}

As an example let us consider tension of an isotropic rectangular plate undergoing PT. The forces $p$ uniformly distributed at the left and right plate boundaries are applied (Figure 2). We assume that in the undeformed state the plate consist of the phase $B$. Under such loading there is an plane deformation state with 
Fig. 2 Tension of a two-phase rectangular plate

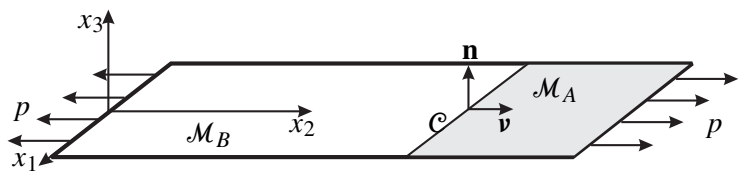

$\mathbf{u}=u_{1} \mathbf{i}_{1}+u_{2} \mathbf{i}_{2}, \boldsymbol{\varphi}=\mathbf{0}$, where $\mathbf{i}_{1}, \mathbf{i}_{2}, \mathbf{i}_{2}$ are the Cartesian base vectors. According to the assumption of the plane deformation, we search for the phase interface $\mathcal{C}$ in the form of the line, which position on $\mathcal{M}$ is given by the equation $x_{1}=\ell(t)$, $0 \leq \ell(t) \leq L$. Hence, $V=d \ell(t) / d t$.

For the one-phase plate there is the following solution

$$
u_{1}=\frac{p}{E h} x_{1}+\text { const, }
$$

where $E$ and $h$ are the Young's modulus and the plate thickness respectively. In the two-phase plate such solution is possible only when $v_{A}=v_{B}=0$, where $v_{A}$ and $v_{B}$ are the Poisson ratios for the bulk material. For the sake of simplicity we follow [15] and assume that $v_{A}=v_{B}=0$. In this case the problem reduces to an one-dimensional problem which is similar to the considered one in [1] or [15].

For $F_{0}=0$, the equilibrium deformation process is described by the path $O A B C$, Figure 3(a). The segment $A B$ describes two-pase state of the plate corresponding the force $p^{\star}$. If we take into account the kinetic equation then in the process of loading the plate deforms according to the path $O A B^{\prime} C$ while for the unloading the plate deforms according to the path $C B A^{\prime} O$. The size of the hysteresis loop $A B^{\prime} A^{\prime}$ depends on $K$ and the loading/unloading velocity. When $K \rightarrow \infty$ the hysteresis loop reduces to the segment $A B$.

When $F_{0} \neq 0$, the plate deforms according to the path $O A_{+} B_{+} C$ while for unloading we have the path $C B_{-} A_{-} O$, Figure 3(b). The size of hysteresis loop becomes larger with the growing value of $F_{0}$. Here growing values of $K$ also lead to decreasing area of the hysteresis loop. For $K \rightarrow \infty$ the limiting paths reduce to

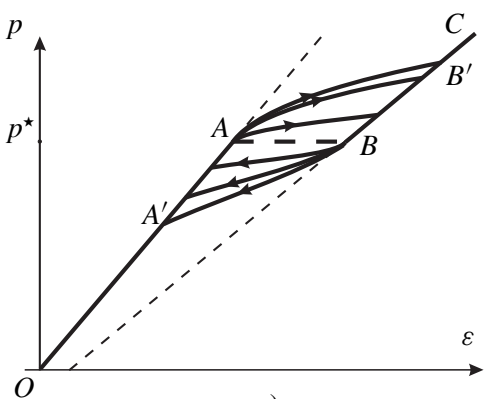

a)

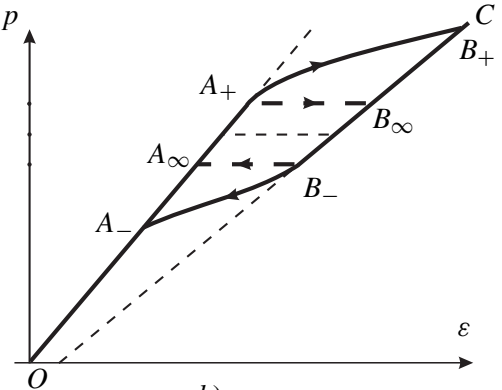

b)

Fig. $3 p-\varepsilon$ curves: a) $F_{0}=0$, b) $F_{0} \neq 0$. 
two different respective segments $A_{+} B_{\infty}$ and $B_{-} A_{\infty}$. Such limit case describes the so-called rate-independent phase transitions $[1,23]$.

Let us note that in the general case $v_{A} \neq v_{B}$ the deformation of the two-pase plate is described by two-dimensional problem. In this case some boundary-layer type solutions may appeared.

\section{Conclusion}

Here we introduced the configurational forces for the plate undergoing phase transformations. These expressions may be also used in the global analysis of a plate with a singular curves of another nature. For example, these approach may be used for modeling of delamination process in a sandwich plate, crack propagation, etc. Indeed, we postulate here the kinetic equation describing the quasistatic motion of singular curve on the base of the consideration of the total energy rate. For example, if one consider a sandwich plate consisting of two parts, delaminated part and undamaged one, then one can introduce a singular curve which separates these parts. Using the concept of the configurational force one may assume the kinetic equation describing the propagation of the delamination curve in the plate.

\section{Acknowledgements}

The authors acknowledge Professors R. Kienzler and A. Berezovski for fruitful discussions and involvement in the field of configurational mechanics.

The second author was supported with the grant of RFBR (07-01-00525) and by the DAAD program "Forschungsaufenthalte für Hochschullehrer und Wissenschaftler" in 2008.

\section{References}

1. Abeyaratne, R. and Knowles, J.K., Evolution of Phase Transitions. A Continuum Theory. Cambridge University Press, Cambridge, 2006.

2. Altenbach, H., An alternative determination of transverse shear stiffnesses for sandwich and laminated plates. Int. J. Solids Struct. 37(25), 2000, 3503-3520.

3. Altenbach, H., On the determination of transverse shear stiffnesses of orthotropic plates. ZAMP 51, 2000, 629-649.

4. Altenbach, H. and Eremeyev, V.A., Direct approach based analysis of plates composed of functionally graded materials. Arch. Appl. Mech. 78(10), 2007, 775-794.

5. Altenbach, H. and Zhilin, P., A general theory of elastic simple shells. Usp. Mekh. 11(4), 1988, 107-148 [in Russian].

6. Altenbach, H. and Zhilin, P.A., The theory of simple elastic shells. In: R. Kienzler, H. Altenbach and I. Ott (Eds.), Critical Review of the Theories of Plates and Shells and New Applications, Lect. Notes Appl. Comp. Mech., Vol. 16. Springer, Berlin, 2004, pp. 1-12. 
7. Berezovski, A., Engelbrecht, J. and Maugin, G.A., Numerical Simulation of Waves and Fronts in Inhomogeneous Solids. World Scientific, New Jersey, 2008.

8. Bhattacharya, K., Microstructure of Martensite: Why It Forms and How It Gives Rise to the Shape-Memory Effect. Oxford University Press, Oxford, 2003.

9. Bhattacharya, K. and James, R.D., A theory of thin films of martensitic materials with applications to microactuators. J. Mech. Phys. Solids 36, 1999, 531-576.

10. Bose, D.K. and Kienzler, R., On material conservation laws for a consistent plate theory. Arch. Appl. Mech. 75, 2006, 607-617.

11. Chróścielewski, J., Makowski, J. and Pietraszkiewicz, W., Statics and Dynamics of Multifold Shells. Non-Linear Theory and Finite Element Method. Wydawnictwo IPPT PAN, Warszawa, 2004 [in Polish].

12. Daly, S., Ravichandran, G. and Bhattacharya, K., Stress-induced martensitic phase transformation in thin sheets of nitinol. Acta Mater. 55, 2007, 3593-3600.

13. Dascalu, C., Maugin, G.A. and Stolz, C. (Eds.), Defect and Material Mechanics. Springer, Dordrecht, 2008.

14. Eremeyev, V. and Pietraszkiewicz, W., The non-linear theory of elastic shells with phase transitions. J. Elasticity 74(1), 2004, 67-86.

15. Eremeyev, V.A. and Pietraszkiewicz, W., Phase transitions in thermoelastic and thermoviscoelastic shells. Arch. Mech. 61(1), 2008, 41-67.

16. Grinfeld, M., Thermodynamics Methods in the Theory of Heterogeneous Systems. Longman, Harlow, 1991.

17. Gurtin, M.E., Thermomechanics of Evolving Phase Boundaries in the Plane. Clarendon-Press, Oxford, 1993.

18. Gurtin, M.E., Configurational Forces as Basic Concepts of Continuum Physics. SpringerVerlag, Berlin, 2000.

19. James, R.D. and Rizzoni, R., Pressurized shape memory thin films. J. Elasticity 59, 2000, 399-436.

20. Kienzler, R. and Herrman, G., Mechanics in Material Space with Applications to Defect and Fracure Mechanics. Springer-Verlag, Berlin, 2000.

21. Kienzler, R. and Herrmann, G., An elementary theory of defective beams. Acta Mech. 62, 1986, 37-46.

22. Kienzler, R. and Herrmann, G., On material forces in elementary beam theory. J. Appl. Mech. 53, 1986, 561-564.

23. Lagoudas, D.C. (Ed.), Shape Memory Alloys. Modeling and Engineering Applications. Springer, Berlin, 2008.

24. Le, K.C., On kinetics of hysteresis. Continuum Mech. Thermodyn. 18, 2007, 335-342.

25. Libai, A. and Simmonds, J.G., The Nonlinear Theory of Elastic Shells, 2nd edn. Cambridge University Press, Cambridge, 1998.

26. Maugin, G.A., Material Inhomogeneities in Elasticity. Chapman Hall, London, 1993.

27. Pieczyska, E.A., Gadaj, S.P., Nowacki, W.K. and Tobushi, H., Phase-transformation fronts evolution for stress- and strain-controlled tension tests in TiNi shape memory alloy. Exp. Mech. 46, 2006, 531-542.

28. Pietraszkiewicz, W., Eremeyev, V.A. and Konopińska, V., Extended non-linear relations of elastic shells undergoing phase transitions. ZAMM 87(2), 2007, 150-159.

29. Steinmann, P. and Maugin, G.A. (Eds.), Mechanics of Material Forces. Advances in Mechanics and Mathematics, Vol. 11. Springer, Berlin, 2006.

30. Zhilin, P.A., Mechanics of deformable directed surfaces. Int. J. Solids Struct. 12, 1976, 635648.

31. Zhilin, P.A., Applied Mechanics. Foundations of the Theory of Shells. St. Petersburg State Polytechnical University, 2006 [in Russian]. 